\title{
MicroRNA-133 Targets Phosphodiesterase IC in Drosophila and Human Oral Cancer Cells to Regulate Epithelial-Mesenchymal Transition
}

\author{
Ji Eun Jung1,2, Joo Young Lee ${ }^{3}$, Hae Ryoun Park ${ }^{1,2,3,4}$, Ji Wan Kang5, Yun Hak Kim6 ${ }^{6}$ Ji Hye Lee ${ }^{1,2,3,4} 4^{\bowtie}$ \\ 1. Department of Life Science in Dentistry, School of Dentistry, Pusan National University, Yangsan 50612, Korea. \\ 2. BK21 FOUR Project, School of Dentistry, Pusan National University, Yangsan 50612, Korea. \\ 3. Dental and Life Science Institute, Pusan National University, Yangsan 50612, Korea. \\ 4. Department of Oral Pathology, School of Dentistry, Pusan National University, Yangsan 50612, Korea. \\ 5. Interdisciplinary Program of Genomic Science, Pusan National University, Yangsan 50612, Korea. \\ 6. Department of Anatomy, Department of Biomedical Informatics, School of Medicine, Pusan National University, Yangsan 50612, Korea. \\ $\triangle$ Corresponding author: E-mail: jihyelee@pusan.ac.kr; Tel.: +82-51-510-8259.
}

() The author(s). This is an open access article distributed under the terms of the Creative Commons Attribution License (https://creativecommons.org/licenses/by/4.0/). See http://ivyspring.com/terms for full terms and conditions.

Received: 2020.11.19; Accepted: 2021.06.24; Published: 2021.07.03

\begin{abstract}
Non-coding microRNAs (miRNAs) have been proposed to play diverse roles in cancer biology, including epithelial-mesenchymal transition (EMT) crucial for cancer progression. Previous comparative studies revealed distinct expression profiles of miRNAs relevant to tumorigenesis and progression of oral cancer. With putative targets of these miRNAs mostly validated in vitro, it remains unclear whether similar miRNA-target relationships exist in vivo. In this study, we employed a hybrid approach, utilizing both Drosophila melanogaster and human oral cancer cells, to validate projected miRNA-target relationships relevant to EMT. Notably, overexpression of dme-miR-133 resulted in significant tissue growth in Drosophila larval wing discs. The RT-PCR analysis successfully validated a subset of its putative targets, including Pde lc. Subsequent experiments performed in oral cancer cells confirmed conserved targeting of human PDEIC by hsa-miR-I33. Furthermore, the elevated level of miR-133 and its targeting of PDEIC was positively correlated with enhanced migrative ability of oral cancer cells treated with LPS, along with the molecular signature of a facilitated EMT process induced by LPS and TGF- $\beta$. The analysis on the RNAseq data also revealed a negative correlation between the expression level of hsa-miR-133 and the survival of oral cancer patients. Taken together, our mammal-to-Drosophila-to-mammal approach successfully validates targeting of PDEIC by miR-133 both in vivo and in vitro, underlying the promoted EMT phenotypes and potentially influencing the prognosis of oral cancer patients. This hybrid approach will further aid to widen our scope in investigation of intractable human malignancies, including oral cancer.
\end{abstract}

Key words: microRNA-133; Drosophila melanogaster; oral cancer; PDE1C; Epithelial-Mesenchymal Transition; mammal-to-Drosophila-to-mammal approach

\section{Introduction}

Since its discovery in early 1990s, microRNA (miRNA) has been at the center of vibrant research activities in the field of human pathology [1]. The function of miRNAs ranges from endogenous post-transcriptional regulators during development to oncomiRs involved in tumorigenesis and progression of various human malignancies, including oral cancer, via downregulation of tumor suppressors. Despite continuous efforts in research, the 5-year survival rate of patients suffering from oral cancer, mostly oral squamous cell carcinoma (OSCC), remains relatively low around $50 \%$ compared to other malignancies [2, 3]. With its incidence rate among the population younger than 45 years fast rising worldwide [4], it requires development of novel therapeutic options with improved efficacy. In an attempt to achieve this goal, a growing body of research efforts has been made to unravel pathologic modulation of miRNAs and their targets that may underlie tumorigenesis and progression of oral cancer. Indeed, recent findings 
address abnormal regulation of miRNAs in disease states from case-control studies or in cancer cell lines in vitro $[5,6]$. With the computational algorithms implemented in the publicly available database, putative targets of a single miRNA can be predicted based upon identification of miRNA-binding sites within the 3'-UTR region of its targets [7-9]. Following prediction, subsequent validation steps of miRNAtarget relationships have been mostly performed in vitro. Thus, whether similar miRNA-dependent targeting occurs in vivo remains largely undetermined. It should also be noted that commonly used target prediction platforms could yield many false positives [10].

Among candidate miRNAs with potential diagnostic and prognostic value for oral cancer, we focus on miR-133 in this study. miR-133 has been one of the most extensively investigated miRNAs for its roles in development of skeletal and heart muscle diseases as well as various types of cancer [11]. miR-133 is known to participate in embryonic myogenesis via targeting Gli3, a transcriptional repressor of the Hedgehog pathway [12] as well as in osteogenesis via targeting Runx2, a bone morphogenetic protein (BMP) response gene crucial for bone formation [13]. Further molecular targets of miR-133 include, but not limited to, epidermal growth factor receptor (EGFR) [14], fibroblast growth factor 1 (FGF1) [15] and insulin-like growth factor-1 receptor (IGF1R) [16]. Regulation of these targets and its functional consequences in tumorigenesis have suggested miR-133 as a potential tumor suppressor in gastric cancer [17], glioma [18] and non-small cell lung cancer [19].

Recent studies indicate a role of miR-133 in cancer progression by regulating the cellular process named epithelial-mesenchymal transition (EMT), a critical phenomenon underlying invasion and migration of cancer cells [20]. Regulation of EMT process by miR-133 was first demonstrated in cardiac myocytes [21]. In their study, Muraoka et al. demonstrated miR-133-dependent cardiac reprogramming by its targeting of Sai1, a key regulator of EMT [21]. Later studies have indicated similar miR-133-dependent regulation of EMT implicated in human cancer. For instance, downregulation of EGFR, Forkhead box C1 (FOXC1) and Forkhead box Q1 (FOXQ1) by miR-133 was thought to mediate reduced cell proliferation, migration and invasion of prostate cancer [14], pituitary adenoma [22] and lung cancer [23], respectively. miR-133dependent targeting of Presenilin 1 (PSEN1) was also linked to the diminished ability of gastric cancer cells to undergo EMT [24]. Meanwhile, there have been only a few studies focused on miR-133-dependent suppression of cancer progression in esophageal squamous cell carcinoma and OSCC, which identified COL1A1 as a presumed target to control invasive and migratory behaviors of cancer cells $[25,26]$. As demonstrated in these studies, however, validation platforms for miR-133 targets in regard to oral cancer and its progression remain mostly restricted to in vitro settings, thus prompting us to unravel additional targets regulated by miR-133 in vivo.

In this study, we introduce an alternative animal model, Drosophila melanogaster, in order to decipher functional relationships between miRNA, specifically miR-133, and its unverified targets in vivo. With high homology to human disease-related genes and relevant signaling pathways of less redundancy [27, 28], Drosophila poses itself as an attractive animal model equipped with sophisticated genetic approaches. We took advantages of this model organism to investigate the relationship between miR-133 and its putative targets in vivo, by forcing its expression in Drosophila larval tissues and by monitoring the transcript level of its putative targets. In order to intensify the clinical significance of our findings in Drosophila, we adopted a hybrid approach to conduct parallel experiments using oral cancer cells in culture. Our findings indicate a shared targeting of phosphodiesterase 1C (PDE1C), a member of phosphodiesterase family, by miR-133 in both Drosophila tissues and human oral cancer cells, underlying promoted tissue growth and more pronounced development of EMT phenotypes. Taken together, our study presents another example of successful application of a mammal-to-Drosophila-tomammal paradigm that allows us to functionally validate human miRNA-dependent regulation of their putative targets. Such combinatorial approach will further help to broaden our understanding of the pathophysiology of oral cancer.

\section{Materials and methods}

\section{Fly stocks}

All crosses and stocks were kept on a standard medium at $24^{\circ} \mathrm{C}$ with humidity between $40 \%$ and $60 \%$. The fly stocks used in our study were acquired from the Bloomington Drosophila Stock Center, including $w^{1118} ;$ P $\{$ UAS-LUC-mir-133.T\} attP2, w*; M\{UAS-mir-133.Sb $\quad Z H-51 D / C y O \quad$ and $w^{*}$; M\{UAS-mir-133.Sb\} ZH-86Fb/TM3, Sb Ser, and the Vienna Drosophila Resource Center for UAS-Pde1c-shRNA (\#101906). The 459.2-GAL4 driver $\left(w^{*} ; \quad P\{G a w B\} 459.2\right)$ was used for expression of UAS-miRNA-133 transgenes specifically in Drosophila imaginal wing discs. 


\section{Image analysis of Drosophila imaginal wing discs}

Imaginal wing discs dissected from the wondering third instar larvae in HL3.1 saline were fixed in PBS containing 3.7\% formaldehyde for 20 minutes and mounted in Vectashield ${ }^{\circledR}$ Antifade Mounting Media (Vector Laboratories, Burlingame, CA, USA). The images were captured with an Olympus microscope (EX51, Olympus, Center Valley, PA, USA) and further processed with Adobe ${ }^{\circledR}$ Photoshop CS6 (Adobe Corporation, San Jose, CA, USA). The ImageJ package (National Institutes of Health (NIH), Bethesda, MD, USA) was used for measurements of overall size of individual wing discs.

For confocal microscopy, individual discs following fixation were permeabilized with PBS containing Triton X-100 and incubated with Alexa Fluor ${ }^{\circledR} 555$ Phalloidin (at 1:20, Cell Signaling Technology) at room temperature for 1 hour before their mounting onto a slide for scanning. The images were taken with a confocal microscope (LSM700, Carl Zeiss, Jena, Germany) and processed with Zen (Carl Zeiss), Image J (NIH) and Adobe ${ }^{\circledR}$ Photoshop CS6 (Adobe Corporation).

\section{Cell culture}

All cells were maintained at $37^{\circ} \mathrm{C}$ in a humidified incubator supplied with $5 \% \mathrm{CO}_{2}$. The human oral squamous cell carcinoma (OSCC) cell lines, including SAS and OSC20, were cultured in Dulbecco's Modified Eagle's Medium and Ham's nutrient mixture F12 (Hyclone, Logan, UT, USA) supplemented with $10 \%$ fetal bovine serum (10\% FBS; GIBCO, ThermoFisher Scientific, Waltham, MA, USA) and penicillin-streptomycin (100 units/ml, Invitrogen, ThermoFisher Scientific). These cells were treated with Plasmocin ${ }^{\mathrm{TM}}$ (InvivoGen, San Diego, CA, USA) for three weeks to remove potential mycoplasma contamination. For stable cell lines expressing miR-133, the pMSCV-puro retroviral vector (Takara Bio Inc, Kusatsu, Japan) was used for subcloning of miR-133. The Phoenix ${ }^{\mathrm{TM}}$ packaging cells (ThermoFisher Scientific) were then transfected with $10 \mu \mathrm{g}$ of the prepared retroviral vector. After being infected with retrovirus, OSCC cells expressing the ectopic miR-133 were selected via an application of puromycin $(2 \mu \mathrm{g} / \mathrm{ml}$, Invitrogen). Stable cell lines were then further treated with Plasmocin ${ }^{\mathrm{TM}}$ (InvivoGen) for three more weeks. Lipopolysaccharides (LPS) derived from a strain of Escherichia coli (E. coli O111:B4; Sigma-Aldrich Inc., St. Louis, MO, USA) was dissolved in DPBS and prepared to a concentration of $5 \mu \mathrm{g} / \mu \mathrm{l}$. Transforming growth factor (TGF)- $\beta 1$ (R\&D Systems, Minneapolis, MN, USA) was dissolved to prepare a stock solution $(20 \mu \mathrm{g} / \mathrm{mL})$ and used at a concentration of $5 \mu \mathrm{g} / \mu \mathrm{l}$. 3-Isobutyl-1methylxanthine (IBMX), a non-selective cAMP/cGMP phosphodiesterase inhibitor, was dissolved and used at a concentration of $1 \mathrm{mM}$. OSCC cell lines initially grown with $10 \%$ FBS were exposed to $0.5 \%$ FBS for 4 hours before 48 hour-long treatments of LPS, TGF- $\beta 1$ or IBMX.

\section{Reverse Transcription Polymerase Chain Reaction (RT-PCR)}

Total RNA was extracted from a total of 5 10 wing discs of the third instar Drosophila larvae using the TRIzol reagent (Invitrogen), followed by a step of cDNA synthesis using TOPscript ${ }^{\mathrm{TM}}$ cDNA synthesis kit (Enzynomics, Inc., Daejeon, Korea). A standard RT-PCR reaction was performed using PrimeScript RT Master Mix (Takara Bio Inc.), according to the manufacturer's instruction. Each RT-PCR reaction was analyzed with gel electrophoresis and visualization using Multiple Gel DOC system (Fuji Photo Film Co., Ltd., Tokyo, Japan).

\section{Real-time quantitative RT-PCR analysis}

The real-time quantitative RT-PCR reaction was performed using TOPreal ${ }^{\mathrm{TM}}$ SYBR Green $\mathrm{qPCR}$ master mix (Enzynomics, Inc.). The reaction cycles consisted of the following steps: 1 cycle of initial denaturation $\left(95^{\circ} \mathrm{C}\right.$ for 10 minutes) and 40 cycles of denaturation $\left(95^{\circ} \mathrm{C}\right.$ for 15 seconds), annealing $\left(60^{\circ} \mathrm{C}\right.$ for 30 seconds) and extension steps $\left(72^{\circ} \mathrm{C}\right.$ for 30 seconds). The quantitative measurement of each transcript was carried out using 7500 Real-Time PCR Instrument System (Applied Biosystems, ThermoFisher Scientific). The mRNA levels of Drosophila actin5c and human 36B4 served as internal controls for normalization in Drosophila and human oral cancer cell experiments, respectively. Each reaction was completed in triplicates. The primer sets used include: Drosophila Pde1c, ATCTTTGTGGACCGCATGTATC as a forward primer and GAACTTGTGAATCG AGCCGTA as a reverse primer; Drosophila actin5c, CCACACCGTCCCCATCTATG as a forward primer and AGTCCAGGGCAACATAGCAC as a reverse primer; human PDE1C-1, TCTCAAAGGATGACTGG AGG as a forward primer and GCTTCTCTGTCAC CCTGTC as a reverse primer; human PDE1C-2, TTCGGCAACATCTCATGGCT as a forward primer and GGAATGGCTGAATTGGCGTC as a reverse primer; human E-cadherin, CATCTTTGTGCCTCCTG AAA as a forward primer and TGGGCAGTGTAG GATGTGAT as a reverse primer; human $\mathrm{N}$-cadherin, CCTGCTTATCCTTGTGCTGA as a forward primer and CCTGGTCTTCTTCTCCTCCA as a reverse primer; human 36B4, CACCATCTTCCAGGAGC 
GAG as a forward primer and GACTCCACGACGTA CTCAGC as a reverse primer.

For quantitative analyses of miR-133 in SAS cells with stable expression of miR-133, total RNA isolated from 6-well plates was converted into cDNA by TaqMan $^{\circledR}$ MicroRNA Reverse Transcription Kit (Applied Biosystems) and quantified by TaqMan ${ }^{\circledR}$ MicroRNA Expression Assays (Applied Biosystems) according to the manufacturer's instructions. Once normalized to the level of $R N U 48$, the relative expression level of miR-133 was determined as fold changes. All TaqMan ${ }^{\circledR}$ MGB probes were purchased from Applied Biosystems (RNU48 and hsa-miR-133a, \#442797S).

\section{Transfection of PDEIC siRNA}

For knockdown of PDE1C in OSCC cells, we designed short-interfering RNAs (siRNAs) with the following sequence: 5137-1 (Human, PDE1C), CUGU CAUCUCCGUUGACUA and UAGUCAACGGAGA UGACAG; 5137-2 (Human, PDE1C), CACAUCAAUC GGGAGAGAU and AUCUCUCCCGAUUGAUGUG; 5137-3 (Human, PDE1C), GACUAGAGCAUACCG GAA and UUCCGGUAUGCUCGUAGUC (Bioneer Co, Ltd, Daejeon, South Korea). The corresponding scrambled siRNA was also purchased from the same source (Bioneer) and used as controls. Transfection of siRNAs was performed using XtremeGENE ${ }^{\mathrm{TM}}$ siRNA Transfection Reagent (Sigma-Aldrich Inc.), at two different concentrations (low and high concentrations of siRNA with $20 \mathrm{ng}$ and $50 \mathrm{ng}$ of total amount, respectively).

\section{Wound-healing assay}

The migratory ability of oral cancer cells was assessed with a scratch wound assay. Briefly, SAS OSCC cells were cultured in six-well plates $\left(2 \times 10^{5}\right.$ cells per well) to $90 \%$ confluence, serum-starved for 4 hours and gently scrapped with a P-200 pipette tip to produce a clean wound area at the center of the cell monolayer. These cells were then treated with LPS at a concentration of $5 \mu \mathrm{g} / \mu \mathrm{l}$ for 24 hours. The wound closure areas were visualized at 0 and 24 hours of incubation under a phase-contrast microscope with a magnification of 100 .

\section{Western blot analysis}

Cells were lysed in RIPA buffer (Elpis-Biotech, Inc., Daejeon, Korea), supplemented with phosphatase inhibitor and protease inhibitor cocktails (Sigma-Aldrich Inc.). The amount of protein in the lysate was measured using Pierce BCA protein assay (ThermoFisher Scientific). Proteins from each lysate were separated on an SDS-PAGE gel and transferred onto nitrocellulose membranes (MilliporeSigma,
Burlington, MA, USA), followed by a blocking step with 3\% BSA. The primary antibodies used include: $\beta$-Actin (Santa Cruz Biotechnology, Dallas, TX, USA), vimentin (D21H3), Slug (c19G7), Snail (C15D3), $\mathrm{N}$-cadherin (D4R1H), E-cadherin (24E10), ZO-1 and claudin-1 (D5H1D) (Cell Signaling Technology, Danvers, MA, USA). All primary antibodies were used at a concentration of 1:1000. The secondary antibodies for chemiluminescence were obtained from Cell Signaling Technology. Chemiluminescence was visualized with WesternBright ${ }^{\mathrm{TM}}$ ECL (Advansta Inc., San Jose, CA, USA) and WesternBright ${ }^{\mathrm{TM}}$ Sirius kit (Advansta Inc.).

\section{Immunofluorescent staining and imaging of OSCC cells}

SAS OSCC cells were plated at a density of $2 \times 10^{4}$ cells per well in an 8-well Lab-Tek II Chambered Coverglas (SPL Life Sciences, Pocheon-si, Korea), incubated for 24 hours and treated with TGF- $\beta 1$ for up to 48 hours. Following incubation, these cells were fixed in PBS containing 4\% paraformaldehyde at room temperature for 15 minutes. Fixed cells were then washed twice, permeabilized with PBS containing Triton X-100 and blocked with 1\% BSA for 1 hour on a locking shaker. The primary antibodies against E-cadherin and claudin-1 (Cell Signaling Technology) were used for imaging at a concentration of 1:100. SAS cells were then incubated with the fluorescein isothiocyanate-conjugated secondary antibody and mounted in Vectashield ${ }^{\circledR}$ Antifade Mounting Medium with DAPI (Vector Laboratories). The images were taken with a confocal microscope (LSM700, Carl Zeiss) and processed with Image J (NIH) and Adobe $^{\circledR}$ Photoshop CS6 (Adobe Corporation).

\section{Gene expression and survival analysis of TCGA collection}

The RSEM normalized RNAseq data and RPKM normalized miRNA data of OSCC were selected from Broad GDAC Firehose (https://gdac.broadinstitute. org/) for subdivisions of TCGA-HNSC according to the following anatomic sites of primary tumors: lip, tongue, floor of mouth, buccal mucosa, hard palate, oropharynx, tonsil, and oral cavity (unspecified). The analyzed dataset consisted of 236 control and 145 OSCC tumor samples. The expression levels of human miR-133s (miR-133a-1 (miR-133a-5p), miR-133a-2 (miR-133a-3p) and miR-133b) and PDE1C in the control and OSCC samples were compared using a Mann-Whitney-Wilcoxon test with Bonferroni correction based on the statannot python package (statannot version 0.2.2 and python version 3.7.1, Python Software Foundation, 2020). Subsequently, the 
survival analyses were performed using the lifelines python package (lifelines version 0.24.0, Python Software Foundation, 2020).

\section{Statistical analysis}

All quantitative RT-PCR experiments each consisting of triplicates were performed at least three times for statistical analyses. The data from two independent groups were compared and analyzed with unpaired two-tailed Student's $t$-test. For multiple comparisons among three groups or more, one-way analysis of variance (ANOVA) was conducted using OriginPro 2020b (OriginLab Corporation, Northampton, MA, USA), followed by Tukey's posthoc tests. For quantification of western blot analyses, one-way ANOVA, non-parametric Kruskal-Wallis ANOVA followed by Dunn's test (both for multiple comparisons among three groups or more), or Mann-Whitney-Wilcoxon test (for two groups) was performed using OriginPro 2020b (OriginLab Corporation). For analyses of the RNAseq datasets, a Mann-Whitney-Wilcoxon test was conducted with Bonferroni correction using the python packages (Python Software Foundation, 2020). The $P$ value less than 0.05 were considered statistically significant.

\section{Results}

\section{Overexpression of miR-133 promoted tissue growth in Drosophila wing discs}

Recent studies have documented differential expression profiles of individual microRNAs (miRNAs) between normal vs. oral cancer tissues as well as oral cancer cell lines, yielding a list of candidates potentially linked to oral cancer, including miR-21, miR-34, miR-133 and miR-203 [29-32]. Among these miRNAs, miR-133 has been mostly proposed as a tumor suppressor implicated in various human malignancies, including oral cancer [17-19, 33, 34]. However, a causative relationship between reduced expression of miR-133 and tumorigenesis or progression of oral cancer has not been well established. In this study, we took advantage of the Drosophila system that would enable monitoring of functional consequence of genetic manipulations in vivo with ease, in addition to the existence of homologous miRNAs with the shared seed sequence. In order to investigate the role of miR-133 in tissue growth, we induced overexpression of miR-133 in Drosophila tissues. The larval imaginal wing discs were selected for this purpose, as they are representative of an epithelial origin, suitable for studying molecular mechanisms underlying the pathophysiology of oral squamous cell carcinoma (OSCC), the most common type of oral cancer. The wing discs have been proven as a useful platform to study a number of cellular signaling pathways important for tumorigenesis and cancer progression, including those involved in the planar cell polarity and the Hedgehog signaling $[35,36]$.

When the sequence of mature Drosophila miR-133 (dme-miR-133-3p) was compared with those of multiple vertebrate miR-133s, including human miR-133s, we found a high degree of conservation (Figure 1A), further confirming the validity of the Drosophila system. Importantly, overexpression of miR-133 in Drosophila wing discs using the GAL4-UAS system [37] resulted in a significant increase in their size, compared to the controls (Figures $1 \mathrm{~B}$ and $1 \mathrm{C}$ ), indicating miR-133 as a factor favoring tissue growth. To examine the nature of promoted growth of the wing discs, we monitored the size of cells constituting the discs. Our results indicated no significant change in the cell size (Supplementary Figure S1), suggesting enhanced cell proliferation corresponding to the increased size of wing discs. It is important to note that our results of miR-133-induced promotion of tissue growth are inconsistent with a proposed role of miR-133 as a tumor suppressor based upon previous clinical and in vitro experimental studies.

\section{Overexpression of $\mathrm{miR}-133$ reduced the transcript level of its predicted target, PDEIC, in both Drosophila wing discs and human oral cancer cells}

Our results on tissue growth following overexpression of miR-133 prompted us to investigate putative targets of miR-133 that might mediate promoted tissue growth in Drosophila wing discs. The putative targets of miR-133 predicted by computational algorithms include an array of molecules ranging from those involved in embryonic myogenesis to key players in cancer biology. For functional validation of miRNA-target relationship in vivo, we first attempted to identify putative targets of miR-133 from TargetScanFly database (release 6.2 and 7.2 in 2012 and 2018, respectively). Upon isolation of putative targets, RT-PCR analyses were performed in larval wing discs overexpressing miR-133 with individual primers specific for each target. Among the candidates screened for changes in their mRNA level, we found a prominent reduction in the amount of Pde1c transcript, with a conserved target site identified within its 3'-UTR region (Figures 2A and 2C). In line with miR-133-dependent downregulation of Pde1c at the level of mRNA, an implementation of RNA interference against $P d e 1 c$ resulted in a modest growth of the wing discs, albeit a lack of statistical significance (Supplementary Figure S2), suggesting a 
partial contribution of miR-133-dependent targeting of Pde1c to promoted tissue growth.

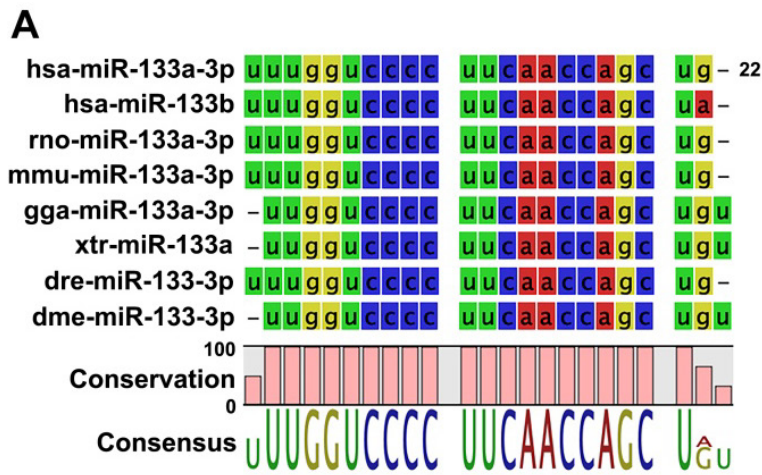

B

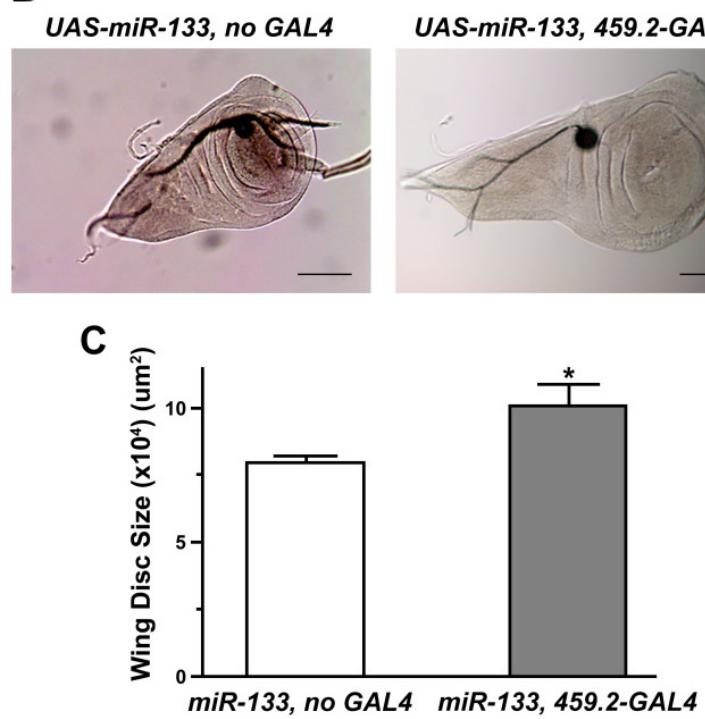

Figure 1. Promoted tissue growth induced by overexpression of Drosophila miR-133. (A) The sequences of mature miRNAs are compared between Drosophila (dme-miR-133) and various mammalian miR-133s (hsa, Homo sapiens; rno, Rattus norvegicus; $m m u$, Mus musculus; gga, Gallus gallus; xtr, Xenopus tropicalis; dre, Danio rerio). Conserved sequences are indicated at the bottom of each comparison. (B) Representative images of Drosophila wing discs are shown for those with (right) and without overexpression of miR-133 (left). Scale bar, $100 \mu \mathrm{m}$. (C) The pooled data are shown for measurements of the size of wing discs in each genotype indicated. The number of wing discs examined: 9 and 18 for UAS-miR-133 with and without 459.2-GAL4, respectively. Mean \pm SEM values are indicated. *, $P<0.05$.

With relatively limited similarity at the level of PDE1C mRNA $(67 \%$ identity with $17 \%$ of gene coverage) and its protein product (58\% identity with $53 \%$ coverage), we investigated the possibility of a conserved targeting of PDE1C transcript by human miR-133 in oral cancer cells, including SAS and OSC20 OSCC cell lines. Importantly, PDE1C was also listed as a putative target of human miR-133 (hsa-miR$133 a-3 p$ and $h s a-m i R-133 b$ ) according to TargetScan Human database (release 6.2 and 7.2 in 2012 and 2018, respectively). The prediction algorithms indeed identified a potential miR-133-binding site within the 3 '-UTR region of human PDE1C (Figure 2B). As the first step of visualizing miR-133-dependent regulation of PDE1C, we monitored the level of endogenous miR-133 in oral cancer cells. Our results indicated extremely low level of miR-133 in OSCC cells, reaching nearly a detection limit of a quantitative RT-PCR analysis (Ct value ranging from 35 to 37). Based upon this finding, we sought to establish cell lines with stable expression of miR-133, thus providing us a better resolution to dissect potential miR-133-dependent regulations of cellular responses in subsequent experiments. When the level of miR-133 was increased nearly 30-fold (Supplementary Figure S3), we found a significant reduction in the level of PDE1C transcripts in these OSCC cells, as confirmed by two independent sets of primers specific for PDE1C (Figure 2D). Together with our findings in Drosophila, these results thus demonstrate a shared targeting of PDE1C by miR-133 in both in vivo and in vitro across different phyla.

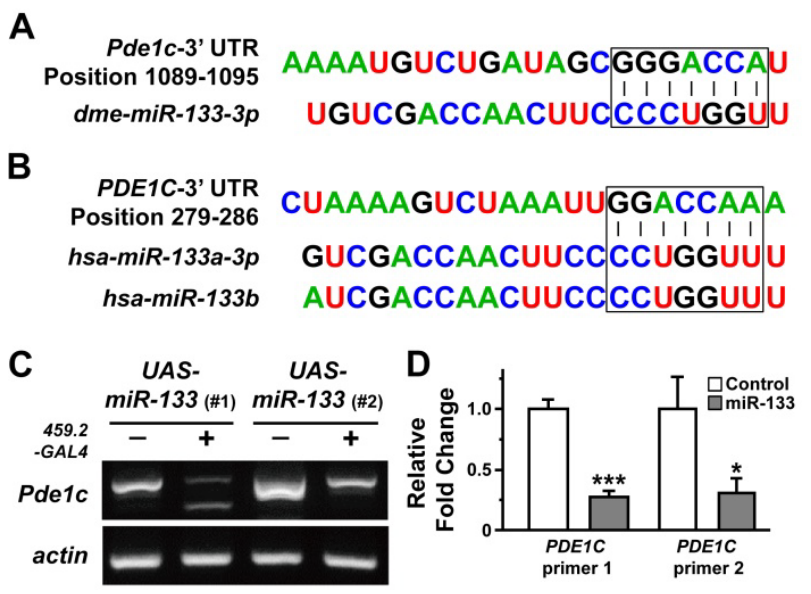

Figure 2. Downregulation of PDEIC by overexpression of miR-133 in both Drosophila wing discs and human oral cancer cells. (A and B) The putative target sites of miR-133 are shown within the 3'-UTR regions of Drosophila (A) and human PDEIC (B). (C) A representative result of RT-PCR reactions is shown to visualize miR-133-dependent downregulation of miR-133 in Drosophila wing discs. Two independent lines of UAS-miR-133 (\#1 and \#2) are used for comparison. (D) The pooled data from quantitative RT-PCR analyses are shown for visualization of miR-133-dependent downregulation of PDEIC in a human oral cancer cell line, SAS cells, with stable expression of miR-133. Two independent sets of primers specific for $P D E I C$ are used for analysis. Mean \pm SEM values are indicated for experiments repeated three times. Two-sample $t$-test is performed. $*, P<0.05$ and $* * *, P<0.001$ for control vs. miR-133.

\section{Overexpression of miR-133 displayed molecular and behavioral signatures representing facilitated epithelial- mesenchymal transition in oral cancer cells}

The involvement of miR-133 in the process of cancer progression has been documented in previous studies on esophageal and oral cancer $[25,26]$, mostly suggesting its role as a factor inhibiting a cellular process named epithelial-mesenchymal transition (EMT) [20]. In the present study, we investigated the effect of miR-133 on molecular networks functioning in the process of EMT in oral cancer cells. We first 
monitored the effect of miR-133 on proliferation of SAS OSCC cells with its stable expression and found no evidence of promoted cell proliferation (Supplementary Figure S4). Next, two independent

A

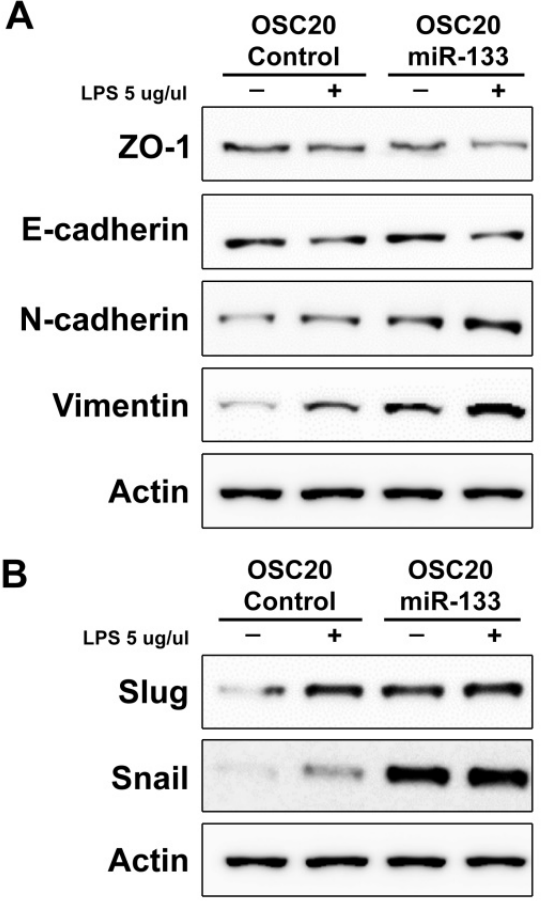

C
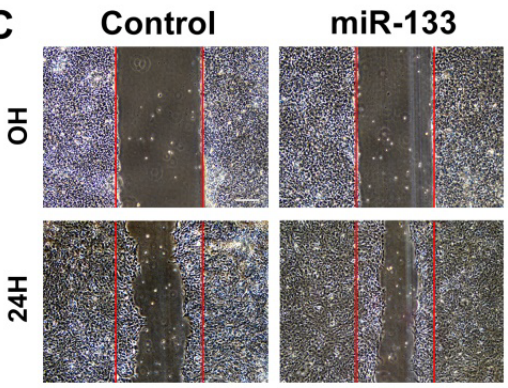

D

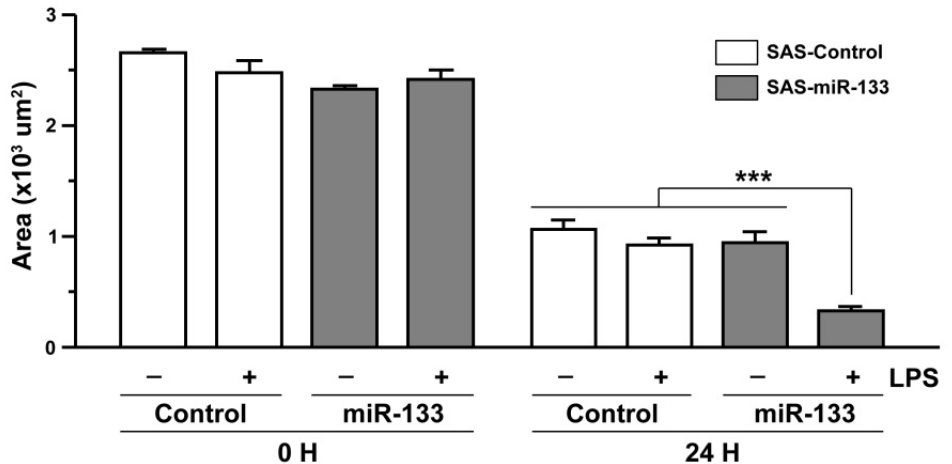

Figure 3. miR-133-dependent facilitation of LPS-induced epithelial-mesenchymal transition. (A and B) The levels of molecular markers characteristic of EMT are indicated by western blot analyses upon an exposure of two independent oral cancer cells, OSC20 (left) and SAS (right) to LPS, in the presence (miR-133) and absence of stable expression of miR-133 (control). (C) The artificially generated gap regions of cultured SAS cell clusters with (miR-133) and without stable expression of miR-133 (control) are shown before $(\mathrm{OH})$ and after 24 hour-long treatments of LPS $(24 \mathrm{H})$. The initial gap generated by scrapping is indicated with red lines in each image. Scale bar, $10 \mu \mathrm{m}$. (D) A quantitative measurement of the wound area is shown for each group before and after a 24 hour-long treatment of LPS. Mean \pm SEM values are indicated for six different regions in cultures. One-way ANOVA test is performed. $* * *, P<0.001$ for comparisons among the experimental conditions indicated.
OSCC cell lines, OSC20 and SAS cells, were treated with lipopolysaccharide (LPS) derived from E. coli in order to facilitate visualization of miR-133-dependent regulation of EMT. A 48 hour-long treatment of LPS was capable of expressing a molecular profile characteristic of EMT [38] (Figure 3). For instance, representative cell surface molecules such as ZO-1 were significantly downregulated, with a similar trend found in E-cadherin as well. In contrast, the expression of mesenchymal cell-biased markers such as N-cadherin and vimentin was significantly promoted in oral cancer cells (Figure 3A). Furthermore, Slug and Snail, two important transcriptional regulators of EMT [39], were significantly upregulated upon an exposure to LPS (Figure 3B), indicating a successful induction of EMT by LPS in oral cancer cells. Importantly, stable expression of miR-133 in these cells appeared to further accelerate phenotype of EMT, both in the presence and absence of LPS, with subtle differences between two oral cancer cell lines tested (Figures 3A and 3B). The quantitative measurement of the protein level from multiple western blot analyses also exhibited similar trends in these EMT probes, albeit with limited statistical significance (Supplementary Figures S5 and S6). These results thus suggest a facilitated EMT process by enhanced expression of miR-133 in oral cancer cells.

The positive correlation between the activation of EMT process and acquisition of invasive ability of cancer cells has been well documented in previous studies (see Son and Moon, 2010, for a review) [40]. With an enhanced EMT phenotype in oral cancer cells upon stable expression of miR-133 (Figures 3A and 3B), we sought to investigate whether such molecular signature would coincide with changes in cellular behaviors. For this purpose, a migratory potential of oral cancer cells was examined with a wound healing assay, through which the width of an artificially generated gap within the cluster of cultured cells was monitored for a specified duration 
of time. While a treatment of OSCC cells with LPS alone did not resulted in a significantly faster closure of the gap, the remaining wound area became significantly narrower in LPS-treated OSCC cells with stable expression of miR-133 (Figures 3C and 3D for quantification), in line with more prominent molecular characteristics of EMT (Figures 3A and 3B; Supplementary Figures S5 and S6). Such behavioral change in cellular migratory potential further confirms a facilitated EMT process and indicates acquisition of more aggressive potential in oral cancer cells with upregulated miR-133.
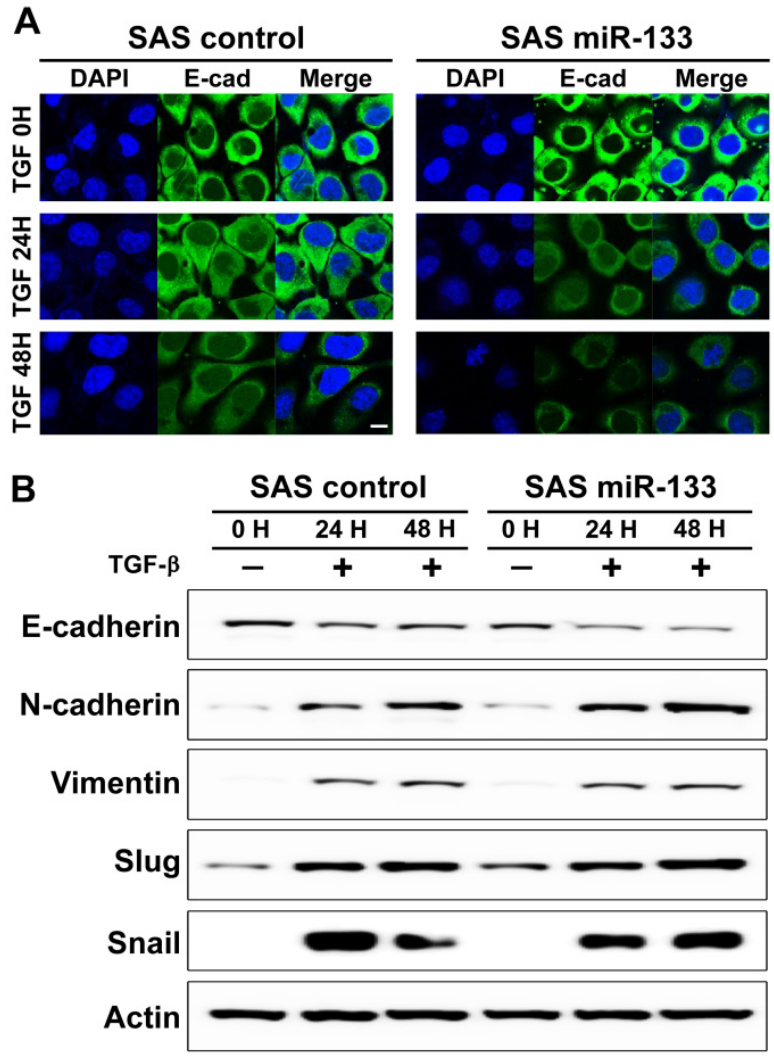

Figure 4. miR-133-dependent facilitation of TGF- $\beta$-induced epithelialmesenchymal transition in oral cancer cells. (A) Representative images of confocal microscopy are shown for visualization of E-cadherin following an exposure of SAS oral cancer cells to TGF- $\beta$ for up to 48 hours, in the presence (miR-133) and absence of stable expression of miR-133 (control). Scale bar, $10 \mu \mathrm{m}$. (B) The levels of molecular markers characteristic of EMT are indicated by a western blot analysis upon an exposure of SAS cells to TGF- $\beta$, in the presence (miR-133) and absence of stable expression of miR-133 (control).

In addition to LPS, an application of transforming growth factor (TGF)- $\beta$ to cancer cells has been mapped to promoted EMT phenotypes in a variety of human malignancies [41], including oral cancer [42]. As expected, we found a prominent downregulation of E-cadherin, an epithelial cellbiased marker, from the boundary of oral cancer cells treated with TGF- $\beta$ (Figure 4A, left panels). A similar trend of downregulation was also evident in the distribution of claudin-1, another well-established epithelial marker (Supplementary Figure S7). Further analysis of a western blot assay confirmed the molecular signature representative of EMT (Figure 4B). Notably, OSCC cells stably expressing miR-133 displayed a more prominent decrease in the immunoreactivity against E-cadherin compared to the controls (Figure 4A, right vs. left panels), suggestive of a facilitated EMT process. Enhanced EMT phenotypes were confirmed with a subsequent western blot analysis, demonstrating significantly reduced expression of E-cadherin in combination with a trend of elevated levels of vimentin, Slug and Snail (Figure 4B; Supplementary Figure S8 for quantitative analyses). It should be noted that there was no evidence of promoted cell proliferation in TGF- $\beta$-treated SAS OSCC cells with stable expression of miR-133, in comparison with the control cells (Supplementary Figure S4), suggesting a minimal to no influence of miR-133 upon proliferative potentials of these cells. Together with our findings in LPS-treated OSCC cells, these results thus imply miR-133 as an important regulator of cancer progression by facilitating the EMT process.

\section{Downregulation of PDEIC was positively correlated with the molecular signature of facilitated EMT in oral cancer cells}

A previous study has suggested a potential implication of PDE1C in regulation of the EMT process in glioblastoma multiform cells [43]. Our results indicating miR-133-dependent downregulation of PDE1C and facilitated EMT phenotypes prompted us to hypothesize the idea that the level of PDE1C would be correlated with development of EMT phenotypes. To test this hypothesis, we designed short-interfering oligonucleotides (siRNAs) against $P D E 1 C$ for its downregulation in OSCC cells. Transfection of three independent PDE1C-siRNAs resulted in effective reduction of its transcript levels (Figure 5A). Interestingly, a subsequent quantitative RT-PCR analysis revealed siRNA-induced changes in the level of E-cadherin and N-cadherin mRNAs (Figure 5B). However, we failed to detect changes at the level of their protein products as well as other EMT markers when visualized with western blot analyses (Supplementary Figure S9), suggesting a relatively limited effect of PDE1C-siRNA itself on regulation of EMT phenotype in the absence of triggering events.

We then investigated whether the level of PDE1C could be positively correlated with development of EMT triggered by a stimulus such as TGF- $\beta$ in OSCC cells (Figure 4). Notably, an application of TGF- $\beta$ to OSCC cells induced a prominent decrease in the level of PDE1C mRNAs, as shown with two independent primers specific for PDE1C (Figure 5C, dotted pattern). A similar 
treatment of TGF- $\beta$ in OSCC cells stably expressing miR-133 displayed a further decrease in the level of PDE1C transcripts (Figure 5C, dark gray), consistent with our data demonstrating a more facilitated EMT phenotype under the same condition (Figure 4B). These data allowed us to postulate a hypothesis that PDE1C might play an important role in mediating the EMT signaling.

Such idea was further supported by our finding demonstrating PDE1C siRNA-induced changes in molecular markers characteristic of EMT. In details, a total of 20 or $50 \mathrm{ng}$ of PDE1C siRNAs, containing a mixture of two independent siRNAs, was sufficient to induce down- and up-regulation of epithelial and mesenchymal markers of EMT, respectively, in the presence of TGF- $\beta$ (Figure 5D). It is also important to note that transfection of a smaller amount of PDE1C siRNAs (20 ng) into TGF- $\beta$-treated OSCC cells was sufficient to induce molecular changes to a degree comparable to those with stable expression of
A

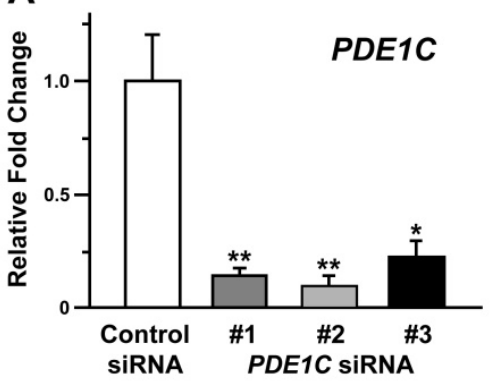

C

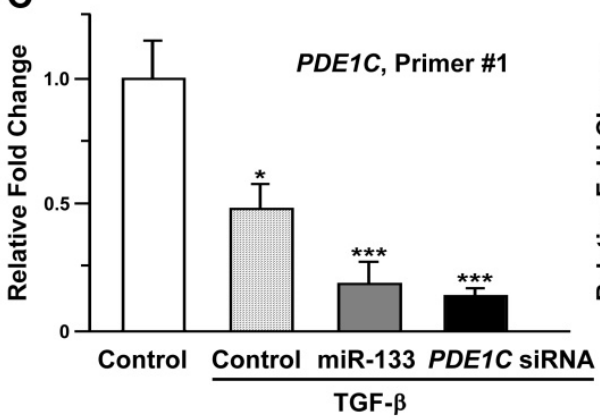

B
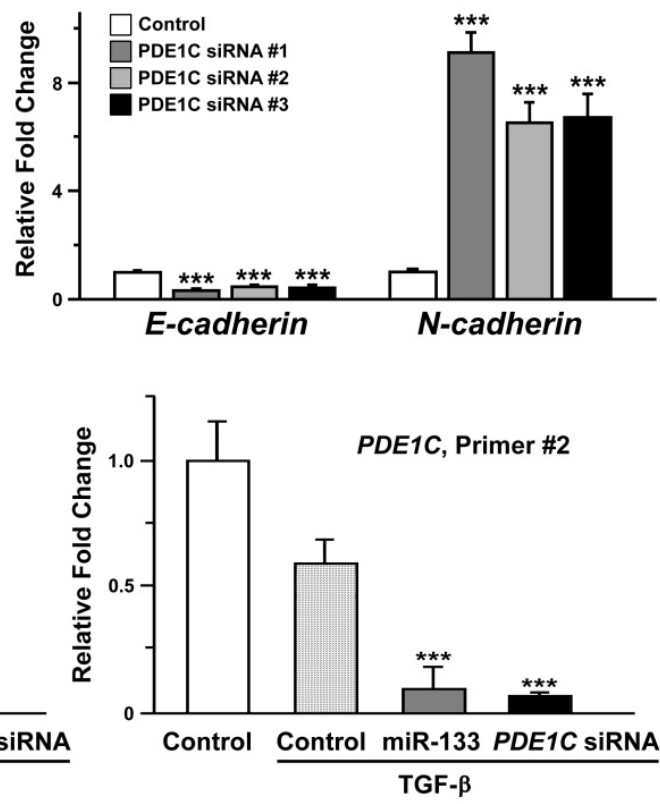

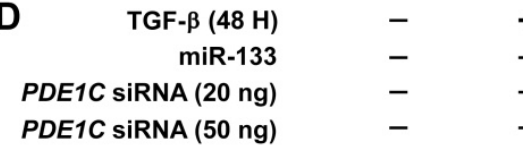

$\begin{array}{llll}+ & + & + & + \\ - & + & - & - \\ - & - & + & - \\ - & - & - & +\end{array}$

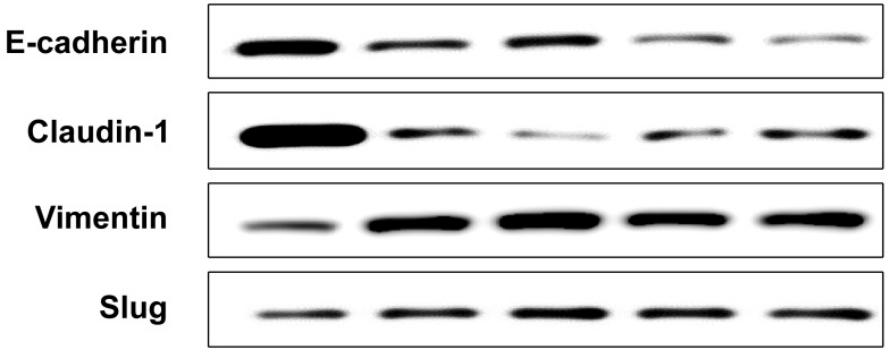

Snai

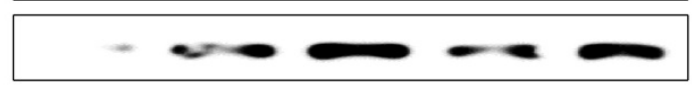

Actin

Figure 5. Facilitated epithelial-mesenchymal transition induced by downregulation of PDE IC in oral cancer cells. (A) The relative levels of PDE IC mRNAs are shown for SAS cells with transfection of three independent short-interfering RNAs (siRNAs) against PDEIC. Mean \pm SEM values are indicated for experiments repeated three times. One-way ANOVA test is performed. *, $P<0.05$ and **, $P<0.01$ for control siRNA vs. other groups. (B) The transcript levels of $E$-cadherin and $N$-cadherin are compared in SAS cells following transfection of three independent siRNAs against PDEIC. Mean \pm SEM values are indicated for experiments repeated three times. One-way ANOVA test is performed. ${ }^{* * *}, P<0.001$ for control siRNA vs. other groups. (C) The transcript levels of PDEIC are measured upon an exposure of SAS cells to TGF- $\beta$, in the presence (miR-133) and absence of stable expression of miR-133 (control) as well as with transfection of PDEIC siRNAs. Two independent sets of primers specific for PDEIC are used for analysis. Mean \pm SEM values are indicated for experiments repeated three times. One-way ANOVA test is performed. *, $P<0.05$ and $* * *, P<0.001$ for control vs. other groups. (D) The levels of molecular markers characteristic of EMT are indicated by a western blot analysis upon an exposure of SAS cells to TGF- $\beta$, in the presence (miR-133) and absence of stable expression of miR-133 (control) as well as with transfection of PDEIC siRNAs at two different concentrations. 
miR-133, implying synergistic effects between miR-133/PDE1C-siRNA and TGF- $\beta$ on EMT (Supplementary Figure S10). To further confirm the relevance of PDE1C in development of EMT phenotype in the presence of TGF- $\beta$, we adopted a pharmacological alternative of PDE1C-siRNA using a non-selective cAMP phosphodiesterase inhibitor, 3-Isobutl-1-methylxanthine (IBMX, $1 \mathrm{mM}$ ). Indeed, an administration of IBMX to TGF- $\beta$-treated OSCC cells revealed profiles of EMT markers similar to those induced by transfection of PDE1C-siRNA (Supplementary Figure S11). Taken together, these data collectively imply a potential regulation of EMT signaling via the activity of a functional network consisting of miR-133 and its target, PDE1C.

\section{Lower expression of miR-133 is correlated with improved survival of human oral cancer patients}

Our findings demonstrating miR-133-dependent facilitation of EMT in human cancer cells raised a possibility that the expression level of miR-133 may be correlated with the prognosis of oral cancer patients. To test this possibility, we analyzed the RNAseq datasets of OSCC selected from Broad GDAC Firehose (https://gdac.broadinstitute.org/) (see Materials and Methods for details). When 236 control and 145 OSCC tumor samples deduced from the collection were analyzed for the expression of miR-133s (miR-133a-1 (miR-133a-5p), miR-133a-2 (miR-133a-3p) and miR-133b), we found a wide range of their expression in both control and OSCC samples, with comparable expression profiles (Figure 6A; $P$-value, 0.578, 0.212 and 0.289 for hsa-miR-133a-1, hsa-miR-133a-2 and
hsa-miR-133b, respectively). However, the subsequent survival analyses revealed consistently improved long-term survival of patients with lower expression of miR-133s (Figure 6B). These clinical data are in line with our findings that suggest a potential role of miR-133 in promoting acquisition of aggressive behaviors of oral cancer cells.

In case of $P D E 1 C$, we found no significant difference in its expression between controls and OSCC patients (Figure 6A; $P$-value, 0.561). While lower expression of PDE1C appeared to favor the survival of some OSCC patients at a later stage, it failed to reach a statistical significance in our hands (Figure 6B; $P$-value, 0.083). In addition, the level of PDE1C was not clearly associated with the survival rate of overall patients at earlier stages following diagnosis (Figure 6B), raising a possibility of miR-133-dependent regulation of unidentified targets other than PDE1C that is likely associated with the prognosis of OSCC patients.

\section{Discussion}

Since its first characterization in early 1900s, Drosophila melanogaster has been serving as a versatile alternative animal model that allowed us to investigate vital functions conserved across phyla in vivo. With the help of sophisticated genetic tools, it continues to expand its horizon in deciphering molecular networks, of which abnormality may underlie a variety of human disease states. In this study, we adopted a hybrid platform that combined Drosophila in vivo and human oral cancer cell-based in vitro systems to investigate functional relationships between a specific miRNA and its target relevant to
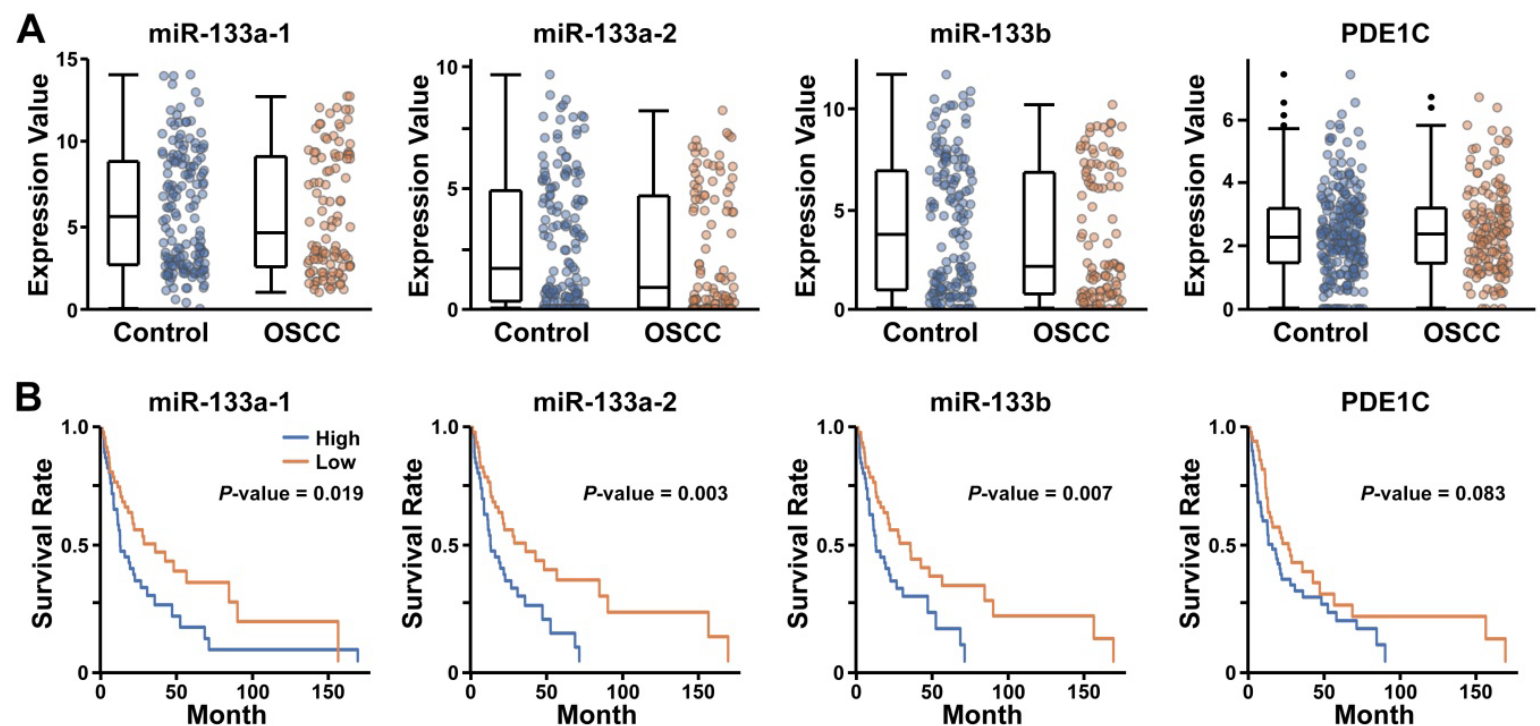

Figure 6. Enhanced survival of oral cancer patients with lower levels of miR-133 and PDE1C. (A) The relative expression levels of three different miR-133s (miR-133a-1 (miR-133a-5p), miR-133a-2 (miR-133a-3p) and miR-133b) and PDEIC are compared between 236 control and 145 OSCC tumor samples deduced from Broad GDAC Firehose (https://gdac.broadinstitute.org/) (see Materials and Methods for details). (B) The survival rates of OSCC patients with differential expression of miR-133s and PDEIC are visualized for comparisons. The significance level of a single analysis is indicated in each plot. 
oral cancer. Validation of miR-133-dependent targeting of PDE1C in both Drosophila wing discs and human oral cancer cells provides a further support to our combinational approach in the process of functional characterization of novel molecules implicated in human diseases. Our results indicate that miR-133-dependent downregulation of PDE1C is likely correlated with promoted tissue growth in Drosophila as well as accelerated expression of EMT phenotypes in oral cancer cells, presumably underlying more pronounced aggressive cellular behaviors.

Previous studies on miR-133 have been mostly centered around its role as a tumor suppressor in subsets of human malignancies, including gastric cancer [17], glioma [18] and non-small cell lung cancer [19]. Furthermore, expression of miR-133 has been frequently linked to reduced cell proliferation and diminished invasive ability of cancer cells via downregulation of EGFR, FOXC1, FOXQ1 and PSEN1 $[14,22-24]$. In line with these data, miR-133dependent upregulation of E-cadherin as a readout of attenuated EMT phenotypes has also been documented in gastric and lung cancer cell lines [23, 24]. Meanwhile, recent studies on esophageal squamous cell carcinoma and OSCC have suggested miR-133-dependent targeting of COL1A1 to control invasive and migratory behaviors of cancer cells, along with significantly reduced cell proliferation and enhanced apoptotic activity induced by miR-133 mimics $[25,26]$. These findings were also coincided with reduced levels of endogenous miR-133 in 20 esophageal and 33 OSCC tumor samples, as well as with a tendency of better prognosis in patients with higher expression of miR-133 [25, 26]. In contrast, our results clearly point out a possibility of miR-133 as an oncomiR, as evidenced by promoted tissue growth in Drosophila wing discs (Figure 1) and by facilitated aggressive cellular behavior in oral cancer cells (Figures 3). Consistently, overexpression of miR-133, presumably via downregulation of $P D E 1 C$, was able to intensify the molecular signature of EMT (Figures 3-5). For instance, we were able to detect downregulation of E-cadherin in OSCC cells with stable expression of miR-133 (Figures 4 and 5; Supplementary Figures S8 and S10), in contrast to the previous findings in gastric and lung cancer cell lines $[23,24]$. It should also be noted that, unlike the aforementioned analyses on esophageal and OSCC samples [25, 26], our bioinformatical analysis of the OSCC RNAseq datasets from Broad GDAC Firehose failed to reveal significant differences in the level of miR-133s (Figure 6A; 236 control and 145 tumor samples). However, it clearly projected a poor survival rate in oral cancer patients with higher expression of miR-133 (Figure 6), thus further in line with the more prominent EMT signatures and acquisition of aggressive behaviors induced by miR-133 in oral cancer cells (Figures 3-5).

While the nature of such discrepancies remains unclear and need to be investigated in detail in future studies, some of them may be attributed to different experimental paradigms employed in each study. For instance, most experimental manipulations of miR-133 in other types of cancer cells, including gastric, lung and esophageal cancer cell lines [23, 24, 26], have relied upon transient transfection of miR-133 mimics, while we sought to establish stable OSCC cell lines with enhanced expression of miR-133. As a result, the level of miR-133 upon induced expression could significantly differ among studies. For instance, Chen et al. [24] and Yin et al. [26] have reported around 3- to 4-fold increases in the level of miR-133 following transfection in gastric and esophageal cancer cell lines, respectively. In contrast, we found nearly a 30-fold increase in OSCC cell lines with stable expression of miR-133 (Supplementary Figure S3). Such difference in the level of miR-133 in each system could influence the activity of miR-133-dependent molecular networks involved in the process of EMT and other relevant cancer biology. It should also be noted that we did not observe any changes in the rate of cell proliferation in OSCC cells with stable expression of miR-133 even in the presence of an EMT-promoting signal such as TGF- $\beta$ (Supplementary Figure S4). This is inconsistent with attenuated cell proliferation and promoted cell death in esophageal and OSCC cells with transient transfection of miR-133 mimics [25, 26], raising a possibility of distinct cellular states examined in these studies. Furthermore, previous studies have identified FOXQ1, PSEN1 and COL1A1 as the major molecular targets of miR-133 underlying its inhibitory role in EMT and cancer progression [23-26]. Here, we propose a novel target of miR-133, PDE1C, at the center of mediating EMT phenotypes, which has been rarely recognized as a critical component in this process. Assuming that the final cellular readout in response to miR-133 would be determined by the collective sum of individual molecular activities each targeted by miR-133, functional consequences of miR-133-dependent regulation may depend upon the level of miR-133 and the choice of its main targets as well as their endogenous activity in a specific cellular context.

As stated above, our results demonstrate a central role of PDE1C in the process of EMT in oral cancer (Figure 5). A previous report on glioblastoma multiforme cells suggested PDE1C as an essential component promoting cell proliferation and invasion 
[43]. In human oral melanoma cell lines, vinpocetine and EHNA, inhibitors of phosphodiesterase 1 (PDE1) and PDE2A, respectively, have been shown to inhibit cell growth [44, 45]. Notably, downregulation of PDE1C by miR-133 in our hands promoted expression of mesenchymal markers in OSCC cells in exchange of downregulation of epithelial cell-biased molecules (Figure 5). Furthermore, our preliminary finding indicates a more pronounced molecular signature of EMT following an administration of IBMX, a cAMP/cGMP phosphodiesterase inhibitor in OSCC cells (Supplementary Figure S11), consistent with the findings in those transfected with PDE1C-siRNA (Figure 5; Supplementary Figure S10). In addition, downregulation of Pde1c in Drosophila wing discs resulted in a noticeable increase in their size via increased cell proliferation (Supplementary Figures S1 and S2), further supporting the idea of PDE1C as an inhibitory signal for cancer progression.

While our data suggestive of accelerated EMT are inconsistent with the previous findings stated above, it should be noted that the activity of PDE1C has never been thoroughly examined in the context of OSCC, the most common form of oral cancer, leaving the functional consequences of its regulation largely unexplored. With the expression profiles and regulatory mechanisms of adenylyl cyclase and PDE diverse among different types of cells, tight regulation of cAMP levels by these enzymes is critical for a wide array of cellular processes, generating the cell- and stimulus-specific responses [46]. It is plausible that the level of endogenous PDE1C could vary to a significant degree among different types of cancer cells. Therefore, a systematic experimental approach will be required in future studies in order to delineate cellspecific, cAMP-dependent regulation of biological responses in different types of cancer cells.

Recent research efforts have been made to discover novel drug candidates targeting PDEs in hopes of developing alternative therapeutic options [47-50]. As a result of such effort, a limited number of PDE inhibitors are currently in clinical use, including PDE3, PDE4 and PDE5 inhibitors [47]. As stated above, our preliminary data indicate pharmacologically induced facilitation of EMT phenotypes in oral cancer cells by an application of a non-selective cAMP/cGMP PDE inhibitor (Supplementary Figure S11). Recently, PDE1 was shown to be specifically targeted by differentiationinducing factor-1 (DIF-1), a Dictyostelium discoideumderived antitumor agent [51]. It will be important to investigate whether treatments of OSCC cells or Drosophila larvae with DIF-1 would lead to similar facilitation of EMT phenotypes or tissue growth observed in our study.
Our findings of miR-133-PDE1C networkdependent regulation of EMT phenotypes in the presence of TGF- $\beta$ raise a possibility of functional crosstalk between miR-133- and TGF- $\beta$-dependent signaling pathways in oral cancer. In this study, we have utilized TGF- $\beta$ as a tool to provide a sensitive background to monitor potential changes in the profile of EMT markers. TGF- $\beta$ is a well-established regulator of transcriptional repressors involved in the expression of EMT phenotypes in a variety of human malignancies [41]. Recent reports have suggested both direct and indirect regulation of TGF- $\beta$ signaling by miRNAs. For instance, the core and downstream signaling components of TGF- $\beta$ such as the ligand and receptors as well as SMADs can be regulated by the activity of various miRNAs, including miR-200 [52, 53]. On the other hand, the activity of downstream effectors of TGF- $\beta$ signaling such as SMADs could influence the biogenesis of miRNAs such as miR-21 via their effects on Drosha-mediated pri-miRNA processing [54, 55]. As a result, these bi-directional interactions constitute functional feedback loops to tightly control the activity of miRNA-TGF- $\beta$ signaling networks [56, 57]. A potential regulation of TGF- $\beta$ signaling by miR-133 has been rarely investigated, with limited numbers of experimental evidence presented in lung cancer cells [23]. Our preliminary results did not indicate consistent alterations in the activity of TGF- $\beta$ signaling pathways, such as phosphorylation of SMADs, in oral cancer cells with relatively high expression of miR-133 (data not shown). Furthermore, a treatment of TGF- $\beta$ did not trigger further expression of miR-133 in oral cancer cells (Supplementary Figure S12). Thus, it remains unclear whether the bidirectional miR-133-TGF- $\beta$ signaling networks function in order to regulate the biology of oral cancer cells. Interestingly, our result indicates TGF- $\beta$-dependent downregulation of PDE1C, as evidenced by a significant decrease in the level of PDE1C mRNA (Figure 5C), in line with a previous report on human alveolar epithelial cells demonstrating TGF- $\beta$-dependent downregulation of PDEs, including PDE1A and PDE3A [58]. Our subsequent analyses revealed a further decrease in PDE1C mRNA upon stable overexpression of miR-133 (Figure 5C), thus suggesting a potential convergence of miR-133- and TGF- $\beta$-dependent signaling upon PDE1C during the process of EMT. The role of such convergence on PDE1C-mediated regulation of EMT in the pathophysiology of oral cancer awaits further investigations.

In summary, we demonstrate miR-133dependent targeting of PDE1C in both Drosophila melanogaster and human oral cancer cells. 
Downregulation of PDE1C by miR-133 is positively correlated with accelerated expression of EMT, a process central to acquisition of aggressive behaviors of cancer cells. Future analysis on the activity regulation of PDE1C in Drosophila and human oral cancer cell platforms will provide a further insight into the molecular mechanisms underlying the pathophysiology of oral cancer.

\section{Abbreviations}

OSCC: oral squamous cell carcinoma; PDEs: Phosphodiesterases; PDE1C: phosphodiesterase type IC; miR-133: microRNA-133; EMT: epithelialmesenchymal transition; LPS: Lipopolysaccharides; TGF- $\beta$ : Transforming growth factor- $\beta$; Gli3: Gliomaassociated Oncogene Family Zinc Finger 3; Runx2: RUNX family transcription factor 2; BMP: Bone morphogenetic protein; EGFR: Epidermal growth factor receptor; FGF1: Fibroblast growth factor 1; IGF1R: Insulin-like growth factor 1 receptor; FOXC1: Forkhead box C1; FOXQ1: Forkhead box Q1; PSEN1: Presenilin 1; EHNA: Erythron-0-(2-hydroxy-3-nonyl) adenine; DIF-1: Differentiation-inducing factor-1.

\section{Supplementary Material}

Supplementary figures.

http://www.jcancer.org/v12p5296s1.pdf

\section{Acknowledgements}

\section{Funding}

This work was supported by the National Research Foundation of Korea (NRF) grants funded by the Ministry of Science and ICT of Korea, NRF-2018R1A5A2023879 (to HR Park and JH Lee) and NRF-2015R1D1A1A02062399 (to JH Lee).

\section{Competing Interests}

The authors have declared that no competing interest exists.

\section{References}

1. Bartel DP. MicroRNAs: genomics, biogenesis, mechanism, and function. Cell. 2004; 116: 281-97.

2. De Angelis R, Sant M, Coleman MP, Francisci S, Baili P, Pierannunzio D, et al. Cancer survival in Europe 1999-2007 by country and age: results of EUROCARE--5-a population-based study. Lancet Oncol. 2014; 15: 23-34.

3. Pignon JP, le Maitre A, Maillard E, Bourhis J, Group M-NC. Meta-analysis of chemotherapy in head and neck cancer (MACH-NC): an update on 93 randomised trials and 17,346 patients. Radiother Oncol. 2009; 92: 4-14.

4. Hussein AA, Helder MN, de Visscher JG, Leemans CR, Braakhuis BJ, de Vet $\mathrm{HCW}$, et al. Global incidence of oral and oropharynx cancer in patients younger than 45 years versus older patients: A systematic review. Eur J Cancer. 2017; 82: 115-27.

5. Soga D, Yoshiba S, Shiogama S, Miyazaki H, Kondo S, Shintani S. microRNA expression profiles in oral squamous cell carcinoma. Oncol Rep. 2013; 30: 579-83.

6. Tran N, McLean T, Zhang X, Zhao CJ, Thomson JM, O'Brien C, et al. MicroRNA expression profiles in head and neck cancer cell lines. Biochemical and Biophysical Research Communications. 2007; 358: $12-7$.

7. Ekimler S, Sahin K. Computational Methods for MicroRNA Target Prediction. Genes (Basel). 2014; 5: 671-83.
8. Friedman RC, Burge CB. MicroRNA target finding by comparative genomics. Methods Mol Biol. 2014; 1097: 457-76.

9. Peterson SM, Thompson JA, Ufkin ML, Sathyanarayana P, Liaw L, Congdon CB. Common features of microRNA target prediction tools. Front Genet. 2014; 5: 23 .

10. Pinzon N, Li B, Martinez L, Sergeeva A, Presumey J, Apparailly F, et al. microRNA target prediction programs predict many false positives. Genome Res. 2017; 27: 234-45.

11. Yu H, Lu Y, Li Z, Wang Q. microRNA-133: expression, function and therapeutic potential in muscle diseases and cancer. Curr Drug Targets. 2014; 15: 817-28

12. Mok GF, Lozano-Velasco E, Maniou E, Viaut C, Moxon S, Wheeler G, et al. miR-133-mediated regulation of the Hedgehog pathway orchestrates embryo myogenesis. Development. 2018; 145.

13. Li Z, Hassan MQ, Volinia S, van Wijnen AJ, Stein JL, Croce CM, et al. A microRNA signature for a BMP2-induced osteoblast lineage commitment program. Proceedings of the National Academy of Sciences of the United States of America. 2008; 105: 13906-11.

14. Tao J, Wu D, Xu B, Qian W, Li P, Lu Q, et al. microRNA-133 inhibits cell proliferation, migration and invasion in prostate cancer cells by targeting the epidermal growth factor receptor. Oncol Rep. 2012; 27: 1967-75.

15. Zhang L, Cheng H, Yue Y, Li S, Zhang D, He R. TUG1 knockdown ameliorates atherosclerosis via up-regulating the expression of miR-133a target gene FGF1. Cardiovasc Pathol. 2018; 33: 6-15.

16. Huang $\mathrm{MB}, \mathrm{Xu} \mathrm{H}, \mathrm{Xie} \mathrm{SJ}, \mathrm{Zhou} \mathrm{H}, \mathrm{Qu}$ LH. Insulin-Like Growth Factor-1 Receptor Is Regulated by microRNA-133 during Skeletal Myogenesis. Plos One. $2011 ; 6$.

17. Liu YF, Zhang $X$, Zhang YJ, Hu ZQ, Yang DJ, Wang CM, et al. Identification of miRNomes in human stomach and gastric carcinoma reveals miR-133b/a-3p as therapeutic target for gastric cancer. Cancer Lett. 2015; 369: 58-66.

18. Liu Y, Han LL, Bai YH, Du W, Yang B. Down-regulation of MicroRNA-133 predicts poor overall survival and regulates the growth and invasive abilities in glioma. Artif Cell Nanomed B. 2018; 46: 206-10.

19. Yang ZQ, Wu CA, Cheng YX. Prognostic Value of microRNA-133a Expression and Its Clinicopathologic Significance in Non-Small Cell Lung Cancer: A Comprehensive Study Based on Meta-Analysis and the TCGA Database. Oncol Res Treat. 2018; 41: 762-8.

20. Bakir B, Chiarella AM, Pitarresi JR, Rustgi AK. EMT, MET, Plasticity, and Tumor Metastasis. Trends Cell Biol. 2020.

21. Muraoka N, Yamakawa H, Miyamoto K, Sadahiro T, Umei T, Isomi M, et al. MiR-133 promotes cardiac reprogramming by directly repressing Snai1 and silencing fibroblast signatures. Embo Journal. 2014; 33: 1565-81.

22. Wang DS, Zhang HQ, Zhang B, Yuan ZB, Yu ZK, Yang T, et al. miR-133 inhibits pituitary tumor cell migration and invasion via down-regulating FOXC1 expression. Genetics and Molecular Research. 2016; 15.

23. Xiao B, Liu HZ, Gu ZY, Ji C. Expression of microRNA-133 inhibits epithelial-mesenchymal transition in lung cancer cells by directly targeting FOXQ1. Arch Bronconeumol. 2016; 52: 505-11.

24. Chen XB, Li W, Chu AX. MicroRNA-133a inhibits gastric cancer cells growth, migration, and epithelial-mesenchymal transition process by targeting presenilin 1. Journal of Cellular Biochemistry. 2019; 120: 470-80.

25. He BX, Lin XZ, Tian FQ, Yu WJ, Qiao B. MiR-133a-3p Inhibits Oral Squamous Cell Carcinoma (OSCC) Proliferation and Invasion by Suppressing COL1A1. Journal of Cellular Biochemistry. 2018; 119: 338-46.

26. Yin YW, Du L, Li XZ, Zhang XY, Gao YL. miR-133a-3p suppresses cell proliferation, migration, and invasion and promotes apoptosis in esophageal squamous cell carcinoma. Journal of Cellular Physiology. 2019; 234: 12757-70.

27. Chien S, Reiter LT, Bier E, Gribskov M. Homophila: human disease gene cognates in Drosophila. Nucleic Acids Res. 2002; 30: 149-51.

28. Pandey UB, Nichols CD. Human disease models in Drosophila melanogaster and the role of the fly in therapeutic drug discovery. Pharmacol Rev. 2011; 63: 411-36.

29. Cao M, Zheng LJ, Liu JZ, Dobleman T, Hu S, Go VLW, et al. MicroRNAs as effective surrogate biomarkers for early diagnosis of oral cancer. Clin Oral Invest. 2018; 22: 571-81.

30. Gorenchtein M, Poh CF, Saini R, Garnis C. MicroRNAs in an oral cancer context - from basic biology to clinical utility. J Dent Res. 2012; 91: 440-6.

31. Perez-Sayans M, Pilar GD, Barros-Angueira F, Suarez-Penaranda JM, Fernandez AC, Gandara-Rey JM, et al. Current trends in miRNAs and their relationship with oral squamous cell carcinoma. J Oral Pathol Med. 2012; 41: 433-43.

32. Scapoli L, Palmieri A, Lo Muzio L, Pezzetti F, Rubini C, Girardi A, et al. MicroRNA expression profiling of oral carcinoma identifies new markers of tumor progression. Int J Immunopathol Pharmacol. 2010; 23: 1229-34

33. Matos LL, Menderico Junior GM, Theodoro TR, Pasini FS, Ishikawa MM, Ribeiro $\mathrm{AAB}$, et al. Cancer-associated fibroblast regulation by microRNAs promotes invasion of oral squamous cell carcinoma. Oral Oncol. 2020; 110: 104909.

34. Citron F, Armenia J, Franchin G, Polesel J, Talamini R, D'Andrea S, et al. An Integrated Approach Identifies Mediators of Local Recurrence in Head and Neck Squamous Carcinoma. Clin Cancer Res. 2017; 23: 3769-80.

35. Hartl TA, Scott MP. Wing tips: The wing disc as a platform for studying Hedgehog signaling. Methods. 2014; 68: 199-206.

36. Olofsson J, Axelrod JD. Methods for studying planar cell polarity. Methods. 2014; 68: 97-104. 
37. Brand AH, Perrimon N. Targeted gene expression as a means of altering cell fates and generating dominant phenotypes. Development. 1993; 118: 401-15.

38. Scanlon CS, Van Tubergen EA, Inglehart RC, D'Silva NJ. Biomarkers of epithelial-mesenchymal transition in squamous cell carcinoma. J Dent Res. 2013; 92: 114-21.

39. Medici D, Hay ED, Olsen BR. Snail and Slug promote epithelial-mesenchymal transition through beta-catenin-T-cell factor-4-dependent expression of transforming growth factor-beta3. Mol Biol Cell. 2008; 19: 4875-87.

40. Son H, Moon A. Epithelial-mesenchymal Transition and Cell Invasion. Toxicol Res. 2010; 26: 245-52

41. Hao Y, Baker D, Ten Dijke P. TGF-beta-Mediated Epithelial-Mesenchymal Transition and Cancer Metastasis. Int J Mol Sci. 2019; 20.

42. Quan J, Elhousiny M, Johnson NW, Gao J. Transforming growth factor-beta1 treatment of oral cancer induces epithelial-mesenchymal transition and promotes bone invasion via enhanced activity of osteoclasts. Clin Exp Metastasis. 2013; 30: 659-70.

43. Rowther FB, Wei WB, Dawson TP, Ashton K, Singh A, Madiesse-Timchou MP, et al. Cyclic nucleotide phosphodiesterase-1C (PDE1C) drives cell proliferation, migration and invasion in glioblastoma multiforme cells in vitro. Mol Carcinogen. 2016; 55: 268-79.

44. Hiramoto K, Murata T, Shimizu K, Morita H, Inui M, Manganiello VC, et al. Role of phosphodiesterase 2 in growth and invasion of human malignant melanoma cells. Cell Signal. 2014; 26: 1807-17.

45. Shimizu K, Murata T, Watanabe Y, Sato C, Morita H, Tagawa T. Characterization of phosphodiesterase 1 in human malignant melanoma cell lines. Anticancer Res. 2009; 29: 1119-22.

46. Sassone-Corsi P. The cyclic AMP pathway. Cold Spring Harb Perspect Biol. 2012; 4

47. Ahmad F, Murata T, Shimizu K, Degerman E, Maurice D, Manganiello V. Cyclic nucleotide phosphodiesterases: important signaling modulators and therapeutic targets. Oral Dis. 2015; 21: e25-50.

48. Desman G, Waintraub C, Zippin JH. Investigation of cAMP microdomains as a path to novel cancer diagnostics. Biochim Biophys Acta. 2014; 1842: 2636-45.

49. Peng $\mathrm{T}$, Gong J, Jin $\mathrm{Y}$, Zhou $\mathrm{Y}$, Tong $\mathrm{R}$, Wei $\mathrm{X}$, et al. Inhibitors of phosphodiesterase as cancer therapeutics. Eur J Med Chem. 2018; 150: 742-56.

50. Savai R, Pullamsetti SS, Banat GA, Weissmann N, Ghofrani HA, Grimminger F, et al. Targeting cancer with phosphodiesterase inhibitors. Expert Opin Investig Drugs. 2010; 19: 117-31.

51. Shimizu K, Murata T, Tagawa T, Takahashi K, Ishikawa R, Abe Y, et al. Calmodulin-dependent cyclic nucleotide phosphodiesterase (PDE1) is a pharmacological target of differentiation-inducing factor-1, an antitumor agent isolated from Dictyostelium. Cancer Res. 2004; 64: 2568-71.

52. Braun J, Hoang-Vu C, Dralle H, Huttelmaier S. Downregulation of microRNAs directs the EMT and invasive potential of anaplastic thyroid carcinomas. Oncogene. 2010; 29: 4237-44.

53. Gregory PA, Bracken CP, Smith E, Bert AG, Wright JA, Roslan S, et al. An autocrine TGF-beta/ZEB/miR-200 signaling network regulates establishment and maintenance of epithelial-mesenchymal transition. Mol Biol Cell. 2011; 22: 1686-98.

54. Davis BN, Hilyard AC, Lagna G, Hata A. SMAD proteins control DROSHA-mediated microRNA maturation. Nature. 2008; 454: 56-61.

55. Davis BN, Hilyard AC, Nguyen PH, Lagna G, Hata A. Smad proteins bind a conserved RNA sequence to promote microRNA maturation by Drosha. Mol Cell. 2010; 39: 373-84

56. Butz H, Racz K, Hunyady L, Patocs A. Crosstalk between TGF-beta signaling and the microRNA machinery. Trends Pharmacol Sci. 2012; 33: 382-93.

57. Suzuki HI. MicroRNA Control of TGF-beta Signaling. Int J Mol Sci. 2018; 19.

58. Kolosionek E, Savai R, Ghofrani HA, Weissmann N, Guenther A, Grimminger $\mathrm{F}$, et al. Expression and activity of phosphodiesterase isoforms during epithelial mesenchymal transition: the role of phosphodiesterase 4. Mol Biol Cell. 2009; 20: 4751-65. 